\title{
ASO Author Reflections: Molecular Testing in Breast Cancer: Is Core Biopsy Equivalent to Surgical Specimen?
}

\author{
Javier I. J. Orozco, MD, and Janie G. Grumley, MD \\ Saint John's Cancer Institute, Providence Saint John's Health Center, Santa Monica, CA
}

\section{PAST}

In the past two decades, gene-expression signatures, perfomed on surgical specimens, have transformed the treatment of breast cancer. Molecular testing adds prognostic and predictive information in patients with hormone receptor-positive, HER2-negative early-stage breast cancer. Notably, the 21-gene recurrence score (OncotypeDx) has allowed for improved selection of patients who would benefit from adjuvant chemotherapy and those who can safely forgo chemotherapy. ${ }^{1}$ Molecular testing, performed on core needle biopsies, has increased in recent years, especially during the COVID-19 pandemic, in the neoadjuvant setting for optimal selection of neoadjuvant systemic therapy. ${ }^{2,3}$ Despite this shift in clinical practice, correlation of the 21-gene recurrence score between paired core needle biopsy and surgical specimens has not been fully explored, raising questions about the equivalence of gene expression profiling in these paired samples.

\section{PRESENT}

In our study, we selected gene expression data, from two publicly available datasets, for paired core needle biopsies and surgical specimens taken from patients with hormonereceptor positive, HER2-negative, early-stage breast cancer ( $n=80$ patients). We evaluated the differences and correlation in the levels of expression levels of the genes included in the 21-gene recurrence score. We also

(C) Society of Surgical Oncology 2021

First Received: 29 June 2021

Accepted: 29 June 2021

Published Online: 4 October 2021

J. G. Grumley, MD

e-mail: Janie.Grumley@ providence.org estimated the recurrence score (also known as microarrayrecurrence score) and evaluated the agreement between the two sample types. ${ }^{4}$

Overall, we found a high correlation in gene expression levels between the core needle biopsy and the surgical specimen, with only minor differences (median difference in gene expressions of the 21-gene approximated zero), demonstrating that both sample types provide similar information. ${ }^{4}$ Furthermore, the concordance rate of the microarray-based 21-gene recurrence score categories was at least $82 \%$, which is similar to the observed concordance rate of anatomopathological evaluation of hormone receptors and HER2 in paired core needle biopsy and surgical specimens. ${ }^{5}$ This provides additional information on the similarities between the diagnostic and surgical tissue samples. However, gene expression did not show absolute concordance, reflecting the presence of some heterogeneity that needs to be considered when making clinical recommendations for systemic therapy.

\section{FUTURE}

Although we found no significant differences in gene expression between the core needle biopsy and surgical specimens, several factors may be involved in the variability of the specimens: the intrinsic tumor heterogeneity, differences in the sampling methods, tissue handling, analytical intra- or interassay variability, and intra- or interobserver interpretation. Therefore, careful handling and standardization of breast tissue specimen processing is critical. This is especially true for the molecular profiling based only in core needle biopsies, since this limited sample may be the only cancer tissue available, particularly in patients achieving a complete pathological response after neoadjuvant systemic therapy. Finally, our study, showing agreement of gene expression signature between the two sample types, needs to be confirmed and validated 
in future studies using testing paired samples with the commercially available molecular platforms before their routine use in clinical practice can be endorsed.

ACKNOWLEDGMENT This study was supported by the Associates for Breast and Prostate Cancer Studies (ABCs) Foundation and the Fashion Footwear Association of New York (FFANY) Foundation.

DISCLOSURES The authors have no conflict of interest disclosures to report.

\section{REFERENCES}

1. Sparano JA, Gray RJ, Ravdin PM, et al. Clinical and genomic risk to guide the use of adjuvant therapy for breast cancer. $N$ Engl $J$ Med. 2019;380(25):2395-405.

2. Iwata H, Masuda N, Yamamoto Y, et al. Validation of the 21-gene test as a predictor of clinical response to neoadjuvant hormonal therapy for ER+, HER2-negative breast cancer: the TransNEOS study. Breast Cancer Res Treat. 2019;173(1):123-33.

3. Bear HD, Wan W, Robidoux A, et al. Using the 21-gene assay from core needle biopsies to choose neoadjuvant therapy for breast cancer: a multicenter trial. J Surg Oncol. 2017;115(8):917-23.

4. Orozco JIJ, Chang SC, Matsuba C, Ensenyat-Mendez M, Grunkemeier GL, Marzese DM, Grumley JG. ASO Visual Abstract: is the 21-gene recurrence score on core needle biopsy equivalent to surgical specimen in early-stage breast cancer?: a comparison of gene expression between paired core needle biopsy and surgical specimens. Ann Surg Oncol. 2021. https://doi.org/10.1245/s10434-021-10589-8

5. Meattini I, Bicchierai G, Saieva C, et al. Impact of molecular subtypes classification concordance between preoperative core needle biopsy and surgical specimen on early breast cancer management: single-institution experience and review of published literature. Eur J Surg Oncol. 2017;43(4):642-8.

Publisher's Note Springer Nature remains neutral with regard to jurisdictional claims in published maps and institutional affiliations. 